\title{
Transmission of light through a single rectangular hole in a real metal
}

\author{
F. J. García-Vidal, ${ }^{1, *}$ L. Martín-Moreno, ${ }^{2}$ Esteban Moreno, ${ }^{1}$ L. K. S. Kumar, ${ }^{3}$ and R. Gordon ${ }^{3, \dagger}$ \\ ${ }^{1}$ Departamento de Física Teórica de la Materia Condensada, Universidad Autónoma de Madrid, E-28049 Madrid, Spain \\ ${ }^{2}$ Departamento de Física de la Materia Condensada, Universidad de Zaragoza-CSIC, E-50009 Zaragoza, Spain \\ ${ }^{3}$ Department of Electrical and Computer Engineering, University of Victoria, Victoria, British Columbia, Canada V8W $3 P 6$
}

(Received 1 August 2006; published 18 October 2006)

\begin{abstract}
A theory is presented to describe the optical transmission through a rectangular hole in a real metal film. The previous theory of the transmission through a rectangular hole in a perfect electric conductor is extended to include the effects associated with having a real metal by adding surface-impedance boundary conditions and an effective index mode calculation. Both the peak and amplitude of the Fabry-Pérot resonance of the fundamental mode agree quantitatively with experiments. Finite-difference time-domain calculations are used to verify the theoretical findings as well as to show the effects of including loss, which is not included in the theory. The localized nature of the transmission resonances is also revealed by analyzing the electric field maps associated with the enhanced transmission process.
\end{abstract}

DOI: 10.1103/PhysRevB.74.153411

PACS number(s): 78.66.Bz, 42.25.Bs, 41.20.Jb, 42.79.Ag

Observations of extraordinary optical transmission through arrays of subwavelength holes in metal films have spurred on intense research activity into understanding and utilizing this phenomenon. ${ }^{1-10}$ Recent works have experimented with the effects on transmission that arise from changing the hole shape. ${ }^{11-13}$ The main findings of those works is that elliptical or rectangular holes can dramatically influence the polarization, the resonance wavelength, and the intensity of the transmission. Random arrays of rectangular holes were used to demonstrate that a resonance exists in the transmission spectrum which is governed by the shape of the individual hole. ${ }^{12}$ The peak wavelength of the resonance could be redshifted by decreasing the width of the short side of the hole. On the other hand, recent studies on isolated rectangular holes have shown the same effects of a resonance in transmission and a redshift that arises when reducing the width of the hole. ${ }^{14}$

To explain how the shape-controlled resonance wavelength arises from a single hole, the influence of the hole shape on the cutoff wavelength of the modes within the hole was considered. ${ }^{15}$ It was shown that the cutoff wavelength increases for a real metal due to increased coupling between evanescent fields on the long edges inside the hole. Later, the origin of the resonance was explained with a theory that incorporated the coupling between the mode inside the hole with the free-space regions on either side of the film. ${ }^{16}$ The physical nature of this effect was attributed to a Fabry-Pérot resonance due to multiple reflections of the mode within the hole at the interfaces with the free-space regions. That theory did not capture the observed redshift phenomenon that arises when the width of the hole is reduced in a real metal because it considered only a perfect electric conductor.

In this paper, a theory that describes quantitatively the transmission resonance for a rectangular hole in a metal film is presented. Here, the previous theory has been generalized to allow for a finite dielectric constant, and thereby captures the new physics associated with having a real metal. Two modifications were required for this generalization: the incorporation of surface-impedance boundary conditions (SIBCs) and the use of the effective index theory to calculate the propagation constant of the fundamental mode within the hole. The redshift in the transmission resonance that arises from decreasing the short-edge width of the hole is demonstrated, and the calculated values agree well with past experiments. Finite-difference time-domain (FDTD) simulations are used to verify the theory, and to show the influence of adding material loss. The small grid-size requirements of the FDTD calculations demonstrate the sensitivity of this resonance effect to evanescent waves at the surface of the metal.

Figure 1(a) shows a schematic of the system under study: a rectangular hole of sides $a_{x}$ and $a_{y}$ perforated on a silver film of thickness $h$. In the calculations presented here, $a_{x}$ $=270 \mathrm{~nm}$ and $h=300 \mathrm{~nm}$ are fixed whereas $a_{y}$ takes several values, $a_{y}=260,185$, and $105 \mathrm{~nm}$. This set of geometrical parameters corresponds to the ones used in the experiments. ${ }^{14}$ The system is illuminated by a normally incident $p$-polarized plane wave of wavelength $\lambda$, the $E$ field pointing along the $y$ direction.

Here we outline the basic theoretical formalism; more details can be found elsewhere. ${ }^{16,17}$ It is based on a modal expansion of the electromagnetic (EM) fields (plane waves in vacuum regions and TE and TM eigenmodes inside the hole). We have shown ${ }^{17}$ that, for subwavelength holes, it is an accurate approximation to consider only the first TE eigenmode. In this way, the EM fields in all regions of space can be written in terms of the modal amplitudes of this mode, $E$ and $E^{\prime}$, at $z=0$ and $h$, respectively. The dielectric properties of a real metal, i.e., the fact that its complex dielectric constant takes finite values, can be incorporated into the formalism in an approximate way by applying SIBCs at the two horizontal interfaces of the structure. This is the first improvement in our theoretical approach. The equations that $E$ and $E^{\prime}$ must satisfy emerge when these SIBCs are applied at the two interfaces of the structure:

$$
(G-\Sigma) E-G_{V} E^{\prime}=I_{0}, \quad-G_{V} E+(G-\Sigma) E^{\prime}=0 .
$$

Note that this system of two linear equations is formally equal to the one found in Ref. 16 where perfect metal boundary conditions were applied. As shown below, the difference with respect to that case resides in the expressions of the magnitudes appearing in it. The nonhomogeneous term $I_{0}$ 

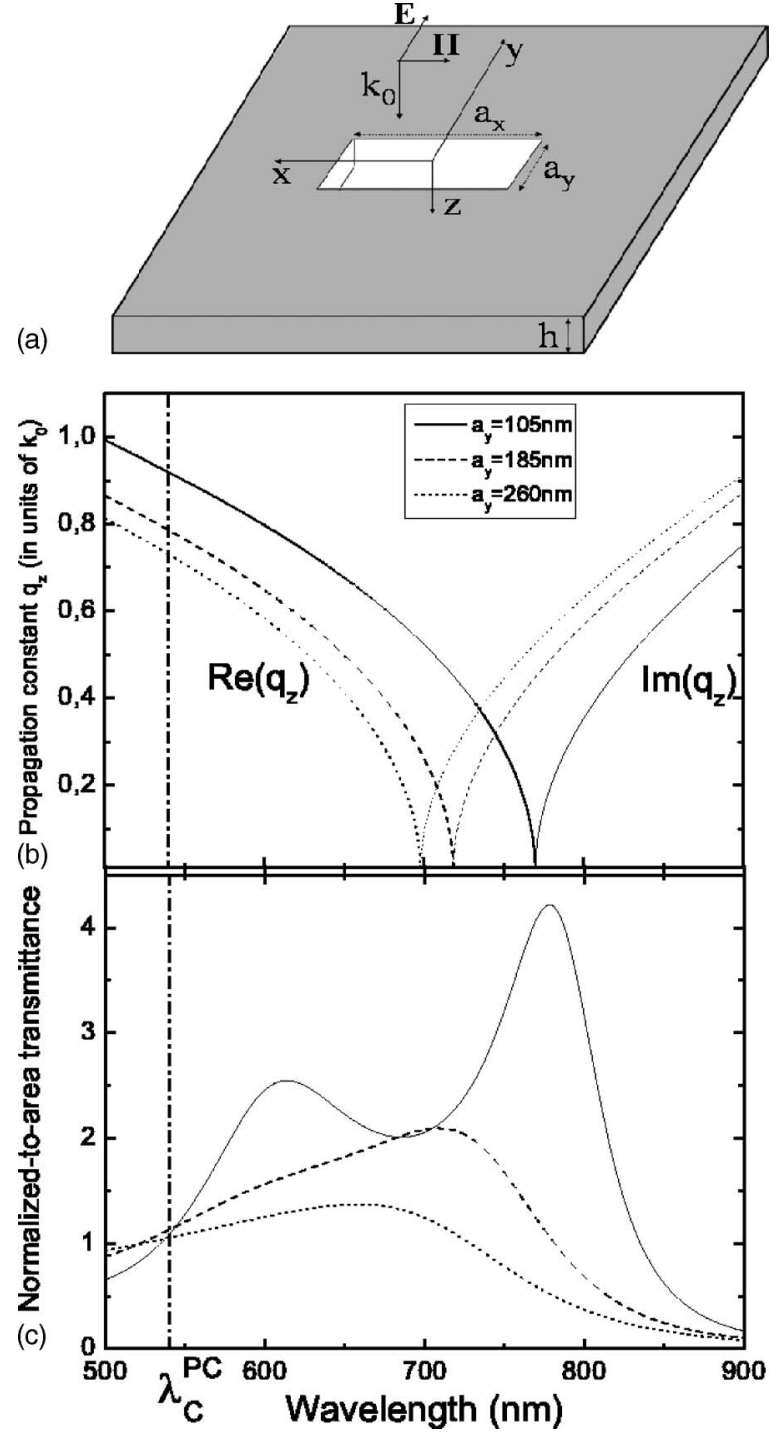

FIG. 1. (a) Diagram of a single rectangular hole of sides $a_{x}$ and $a_{y}$ perforated on a silver film of thickness $h$. The structure is illuminated by a normally incident $p$-polarized plane wave with its $E$-field vector pointing along the $y$ axis. (b) Real and imaginary parts of the propagation constant $q_{z}$ for rectangular holes with $a_{x}$ $=270 \mathrm{~nm}$ and $a_{y}=105,185$, and $260 \mathrm{~nm}$ perforated on a silver film with $h=300 \mathrm{~nm}$. (c) Normalized-to-area transmittance $(T)$ versus $\lambda$ for the three cases displayed in (b) calculated with the modal expansion technique.

measures the overlap between the incident plane wave and the TE eigenmode inside the hole. For normally incident radiation, $I_{0}=4 \sqrt{2} /\left[i \pi\left(1+Z_{S}\right)\right]$, where $Z_{S}$ is the surface impedance of the metal, $Z_{S}=1 / \sqrt{\epsilon(\lambda)}, \epsilon(\lambda)$ being the $\lambda$-dependent dielectric constant of silver. In our calculations, we have used the values for $\epsilon$ as tabulated in Ref. 18. The coupling between the two sides of the hole is accounted for by the term $G_{V}$ and is basically controlled by the propagation constant of the fundamental TE mode, $q_{z}$, and by its admittance $Y_{\mathrm{TE}}=q_{z} / k_{\omega}\left[\left(\right.\right.$ with $\left.\left.k_{\omega}=2 \pi / \lambda\right)\right]$ :

$$
G_{V}=\frac{2 i Y_{\mathrm{TE}}}{e^{i q_{z} h}\left(1+Z_{S} Y_{\mathrm{TE}}\right)^{2}-e^{-i q_{z} h}\left(1-Z_{S} Y_{\mathrm{TE}}\right)^{2}} .
$$

The term $\Sigma$ in Eq. (1) also depends on $q_{z}$ and $Y_{\mathrm{TE}}$ :

$$
\Sigma=i Y_{\mathrm{TE}} \frac{e^{i q_{z} h}\left(1+Z_{S} Y_{\mathrm{TE}}\right)+e^{-i q_{z} h}\left(1-Z_{S} Y_{\mathrm{TE}}\right)}{e^{i q_{z} h}\left(1+Z_{S} Y_{\mathrm{TE}}\right)^{2}-e^{-i q_{z} h}\left(1-Z_{S} Y_{\mathrm{TE}}\right)^{2}} .
$$

The self-illumination of the hole, via vacuum modes, is controlled by $G$, which for this particular hole geometry can be expressed as

$$
\begin{aligned}
G= & \frac{i a_{x} a_{y}}{8 \pi^{2} k_{\omega}} \int_{-\infty}^{+\infty} \int_{-\infty}^{+\infty} d k_{x} d k_{y} \frac{k_{\omega}^{2}-k_{x}^{2}}{k_{z}} \frac{k_{\omega}}{k_{\omega}+Z_{S} k_{z}} \operatorname{sinc}^{2}\left(\frac{k_{y} a_{y}}{2}\right) \\
& \times\left[\operatorname{sinc}\left(\frac{k_{x} a_{x}+\pi}{2}\right)+\operatorname{sinc}\left(\frac{k_{x} a_{x}-\pi}{2}\right)\right]^{2}
\end{aligned}
$$

where $k_{z}=\sqrt{k_{\omega}^{2}-k^{2}}$ and $k^{2}=k_{x}^{2}+k_{y}^{2}$. Notice that the expressions for $I_{0}, G_{V}, \Sigma$, and $G$ in a perfect conductor film given in Ref. 16 can be easily obtained from Eqs. (2)-(4) by just setting $Z_{S}=0$.

The second improvement of this theory is to incorporate a realistic value for the propagation constant of the TE mode $\left(q_{z}\right)$ inside the hole. For a perfect electric conductor $q_{z}$ $=\sqrt{k_{\omega}^{2}-\left(\pi / a_{x}\right)^{2}}$. By using the effective index method, ${ }^{15}$ an analytic expression for $q_{z}$ in a real metal can be derived which gives very accurate results when compared with finitedifference calculations. Within this approach, although the absorption in the metal is neglected, both the penetration of the EM fields inside the metal and the existence of surface plasmons are captured into $q_{z}$.

Figure 1(b) shows the effective index calculations of the real and imaginary parts of $q_{z}$ versus $\lambda$ rendered for rectangular holes with fixed $a_{x}=270 \mathrm{~nm}$ and three different values of $a_{y}=105,185$, and $260 \mathrm{~nm}$. In the three cases, the cutoff wavelength $\lambda_{C}$ (defined as the wavelength at which $\left.q_{z}=0\right)$ is much longer than the perfect conductor value $\lambda_{C}^{\mathrm{PC}}=2 a_{x}$ $=540 \mathrm{~nm}$. Importantly, the shift to longer wavelengths increases when $a_{y}$ decreases.

Figure 1(c) shows the normalized-to-area transmission $(T)$ spectra for the three cases analyzed in Fig. 1(b). These spectra have been computed by using the formalism described above. It is clear that there is a close correlation between the locations of the transmission resonances and the values of $\lambda_{C}$ for the three rectangular holes analyzed. For the case of a rectangular hole perforated on a perfect conductor, we also demonstrated that a transmission resonance emerges at the corresponding cutoff wavelength, $\lambda_{C}^{\mathrm{PC}} \cdot{ }^{16}$ These combined results allow us to raise a general conclusion: for a rectangular hole drilled on a metal, there is a transmission resonance located at the cutoff wavelength, associated with the condition $q_{z}=0$. In a real metal (finite $\epsilon$ ), due to the different effective propagation characteristics of the fundamental TE mode, this cutoff wavelength is shifted to longer wavelengths with respect to the perfect conductor case $(\epsilon=-\infty)$. However, our results suggest that the physical origin of the transmission resonances appearing in both real metals and perfect conductors is the same. Figure 1(c) also shows the redshift in the transmission peak wavelength with reducing short-edge width of the hole and that $T$ increases with the ratio $a_{x} / a_{y}$. These two trends agree very well with past experiments. ${ }^{14}$ 


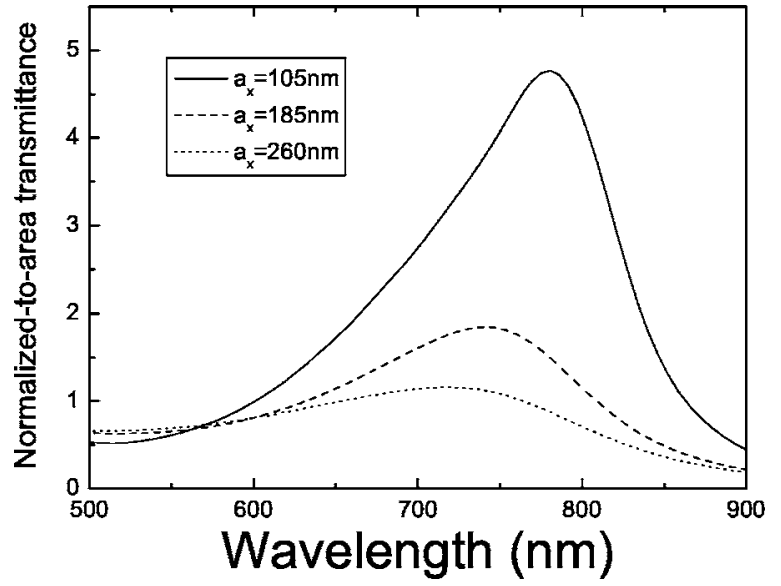

FIG. 2. $T$ for a normally incident plane wave versus $\lambda$ as obtained with the FDTD numerical code for the same three cases analyzed in Fig. 1(c).

To further corroborate the theory and to consider the effects of absorption in the metal, we have conducted FDTD numerical simulations on these structures. Convergence of the transmission spectra was found by varying the grid sizes from 20 to $3 \mathrm{~nm}$. This last value for the grid size ensures that the surface plasmon effects are accurately captured. The source used was a plane wave with a center wavelength of $700 \mathrm{~nm}$ with a broadband pulse of $5 \mathrm{fs}$.

Figure 2 shows $T$ versus $\lambda$ obtained with the FDTD code for the three cases analyzed in Fig. 1(c). Remarkably, there is a very good agreement between the two very distinct theoretical approaches on the location of the resonances and on their transmission values. The main difference is linked to the appearance of an additional peak located at around $600 \mathrm{~nm}$ in the modal expansion calculations (clearly evident in the $a_{y}=105 \mathrm{~nm}$ case and showing lower amplitude in the other two cases). FDTD calculations in which the absorption in the metal is gradually reduced indicate that the origin of this discrepancy is related to neglecting silver absorption on the vertical walls of the hole within the modal expansion formalism. Except for this feature, the two theoretical spectra closely resemble the experimental ones (see Fig. 3c in Ref. 14), allowing us to safely conclude that the physical origin of the transmission peaks found in isolated rectangular holes perforated in real metals stems from resonant modes that present a nearly zero propagation constant $\left(q_{z}=0\right)$. At this condition, the group velocity of the mode tends to zero, resulting in a huge increase of the photonic density of states.

This conclusion is reinforced by looking at the amplitude of the $E$ field on two different planes ( $x=0$, top panel, and $z=0^{-}$, bottom panel, in Fig. 3) for the case $a_{x}=270 \mathrm{~nm}$ and $a_{y}=105 \mathrm{~nm}$ evaluated at the resonant wavelength $(\lambda$ $=780 \mathrm{~nm})$ using the FDTD code. The top panel shows that the $E$-field amplitude is practically uniform inside the hole, as corresponds to a mode with $q_{z}=0$. The bottom panel reveals the localized character of the mode. It is worth noticing the similarities of these pictures with the ones reported for a
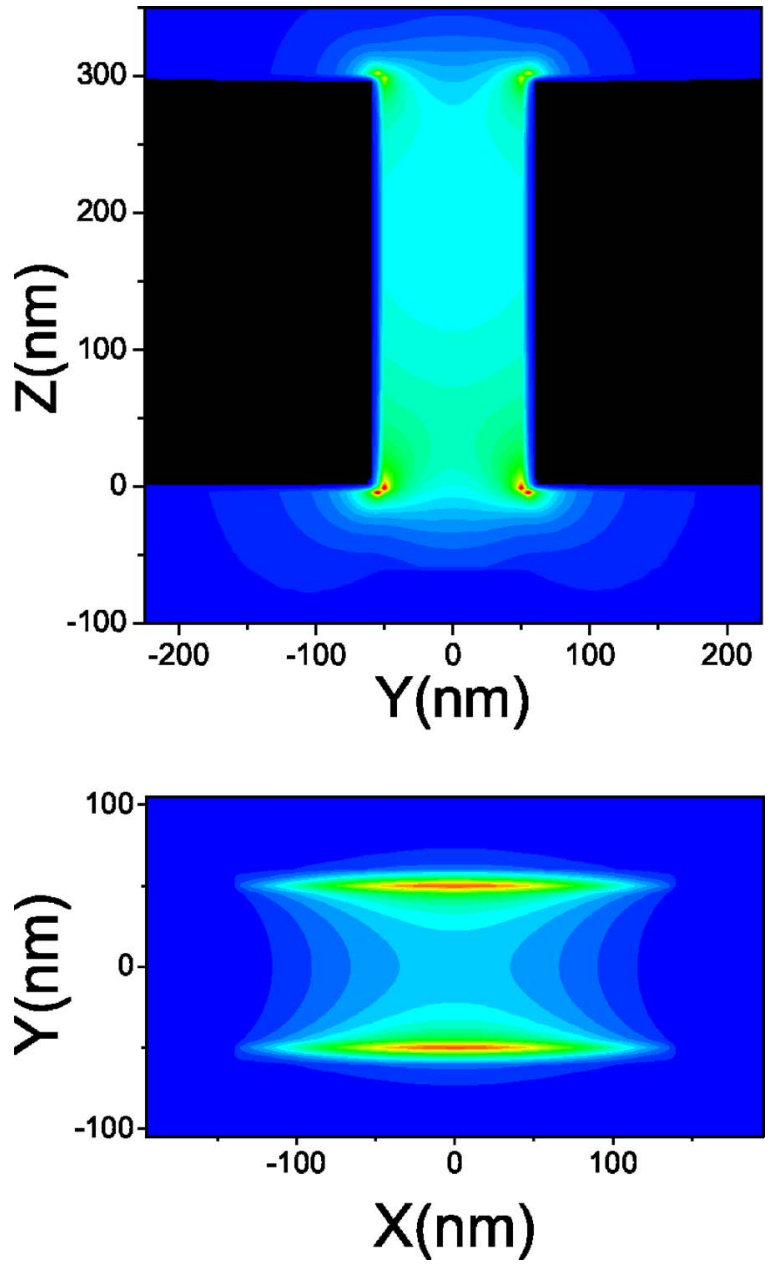

FIG. 3. (Color online) $E$-field amplitude evaluated at the resonant wavelength for a rectangular hole with $a_{x}=270 \mathrm{~nm}$ and $a_{y}$ $=105 \mathrm{~nm}$ in two different planes: cut through the center of the rectangle ( $x=0$, top panel; wave impinging from the bottom) and entrance surface $\left(z=0^{-}\right.$, bottom panel).

single rectangular hole perforated on a perfect conductor. ${ }^{16}$

In conclusion, we have demonstrated theoretically that single rectangular holes perforated on real metals exhibit transmission resonances that appear near the wavelength in which the propagation constant inside the hole waveguide approaches zero. The physical origin of these resonances is very similar to the one previously found for single rectangular holes perforated on perfect conductors, except for the redshift in the resonance peak resulting from the change in the cutoff wavelength. Good quantitative agreement was found with past experiments and FDTD calculations. The FDTD calculations also show the effects of including losses, which were not present in the theory.

Financial support by the Spanish MCyT under Contract No. MAT2005-06608-C02, and the EC under Projects No. FP6-2002-IST-1-507879 and No. FP6-NMP4-CT-2003505699 is gratefully acknowledged. 
*Electronic address: fj.garcia@uam.es

†lectronic address: rgordon@uvic.ca

${ }^{1}$ T. W. Ebbesen, H. J. Lezec, H. F. Ghaemi, T. Thio, and P. A. Wolff, Nature (London) 391, 667 (1998).

${ }^{2}$ L. Martin-Moreno, F. J. Garcia-Vidal, H. J. Lezec, K. M. Pellerin, T. Thio, J. B. Pendry, and T. W. Ebbesen, Phys. Rev. Lett. 86, 1114 (2001).

${ }^{3}$ J. B. Pendry, L. Martin-Moreno, and F. J. Garcia-Vidal, Science 305, 847 (2004).

${ }^{4}$ M. Beruete, M. Sorolla, I. Campillo, J. S. Dolado, L. MartinMoreno, J. Bravo-Abad, and F. J. Garcia-Vidal, Opt. Lett. 29, 2500 (2004).

${ }^{5}$ A. P. Hibbins, B. R. Evans, and J. R. Sambles, Science 308, 670 (2005).

${ }^{6}$ E. S. Kwak, J. Henzie, S. H. Chang, S. K. Gray, G. C. Schatz, and T. W. Odom, Nano Lett. 5, 1963 (2005).

${ }^{7}$ H. Shin, P. B. Catrysse, and S. Fan, Phys. Rev. B 72, 085436 (2005).

${ }^{8}$ P. Lalanne, J. C. Rodier, and J. P. Hugonin, J. Opt. A, Pure Appl. Opt. 7, 422 (2005).

${ }^{9}$ J. V. Coe, S. M. Williams, K. R. Rodriguez, S. Teeters-Kennedy,
A. Sudnitsyn, and F. Hrovat, Anal. Chem. 78, 1384 (2006).

${ }^{10}$ J. Bravo-Abad, A. Degiron, F. Przybilla, C. Genet, F. J. GarciaVidal, L. Martin-Moreno, and T. W. Ebbesen, Nat. Phys. 2, 120 (2006).

${ }^{11}$ R. Gordon, A. G. Brolo, A. McKinnon, A. Rajora, B. Leathem, and K. L. Kavanagh, Phys. Rev. Lett. 92, 037401 (2004).

${ }^{12}$ K. J. Klein Koerkamp, S. Enoch, F. B. Segerink, N. F. van Hulst, and L. Kuipers, Phys. Rev. Lett. 92, 183901 (2004); K. L. van der Molen, K. J. Klein Koerkamp, S. Enoch, F. B. Segerink, N. F. van Hulst, and L. Kuipers, Phys. Rev. B 72, 045421 (2005).

${ }^{13}$ H. Cao and A. Nahata, Opt. Express 12, 3664 (2004).

${ }^{14}$ A. Degiron, H. J. Lezec, N. Yamamoto, and T. W. Ebbesen, Opt. Commun. 239, 61 (2004).

${ }^{15}$ R. Gordon and A. G. Brolo, Opt. Express 13, 1933 (2005).

${ }^{16}$ F. J. Garcia-Vidal, E. Moreno, J. A. Porto, and L. Martin-Moreno, Phys. Rev. Lett. 95, 103901 (2005).

${ }^{17}$ J. Bravo-Abad, F. J. Garcia-Vidal, and L. Martin-Moreno, Phys. Rev. Lett. 93, 227401 (2004).

${ }^{18}$ Handbook of Optical Contants of Solids, edited by E. D. Palik (Academic, Orlando, FL, 1985). 